\title{
Doubling the field of view in off-axis low-coherence interferometric imaging
}

\author{
Pinhas Girshovitz and Natan T Shaked
}

We present a new interferometric and holographic approach, named interferometry with doubled imaging area (IDIA), with which it is possible to double the camera field of view while performing off-axis interferometric imaging, without changing the imaging parameters, such as the magnification and the resolution. This technique enables quantitative amplitude and phase imaging of wider samples without reducing the acquisition frame rate due to scanning. The method is implemented using a compact interferometric module that connects to a regular digital camera, and is useful in a wide range of applications in which neither the field of view nor the acquisition rate can be compromised. Specifically, the IDIA principle allows doubling the off-axis interferometric field of view, which might be narrower than the camera field of view due to low-coherence illumination. We demonstrate the proposed technique for scan-free quantitative optical thickness imaging of microscopic biological samples, including live neurons and a human sperm cell in rapid motion under high magnification. In addition, we used the IDIA principle to perform non-destructive profilometry during a rapid lithography process of transparent structures.

Light: Science \& Applications (2014) 3, e151; doi:10.1038/lsa.2014.32; published online 14 March 2014

Keywords: cell imaging; holography; interferometry; lithography; quantitative optical microscopy

\section{INTRODUCTION}

Digital interferometric and holographic imaging is a quantitative optical imaging technique that is able to capture the complex wavefront (amplitude and phase) of light interacting with a sample. This capturing is performed by recording the spatial interference pattern of the beam that interacted with the sample and a mutually coherent reference beam using a digital camera. Interferometric and holographic imaging techniques have affected many fields of science over the past 55 years. One of these techniques, developed mostly in the past 10 years, is digital interferometric phase microscopy (IPM), also called digital holographic microscopy. IPM is a label-free quantitative tool for capturing the complex wavefront of transparent or translucent microscopic samples, and processing it into the spatial optical thickness maps of the samples. This tool is useful for a wide range of applications, including label-free biological cell imaging and nondestructive quality tests of optical elements. ${ }^{1-7}$

Although it is easier to obtain interference with a highly coherent source, using such a source in interferometric and holographic imaging in general, and in IPM in particular, significantly reduces the image quality due to parasitic interferences and coherent noise. $^{7-12}$ To overcome this problem, low-coherence light sources are employed. However, to obtain interference using these sources, meticulous alignment between the optical paths of the two beams is required. For off-axis interferometric geometry, which enables a single-exposure acquisition mode, the sample and the reference beams interfere on the digital camera with a small angle. Thus, even with strict alignment between the beam optical paths, it is frequently not possible to obtain low-coherence interference on the entire camera field of view (FOV) due to the angular beam-path difference across the beam cross-section, which might be above the coherence length of the source. ${ }^{9}$ The practical implication of this limitation is that large samples cannot be simultaneously recorded by off-axis interferometry on the entire camera sensor using low-coherence sources. Diffractive gratings can solve this problem by tilting the field of the beams to be in plane, ${ }^{9-13}$ with the cost of possible aliasing and image modulation.

Even when using a highly coherent source, where the off-axis interference is obtained on the entire camera sensor, many interferometric setups have a small acquisition FOV, because the size of the camera sensor used for the digital recording of the interferogram is smaller than the optical FOV defined by the microscope-objective aperture projected onto the camera plane. This narrow camera-FOV restriction is especially critical when imaging large samples with fine details under high magnifications, or when imaging dynamics of objects that might move out of the camera FOV.

In this paper, we present a new technique, referred to as interferometry with doubled imaging area (IDIA), which is capable of doubling the off-axis interferometric FOV without using special optical elements. This FOV doubling is performed by optically multiplexing two off-axis orthogonal interferograms from different FOVs of the sample on the same camera sensor using a smart design of the optical system. In the case that the off-axis interference is not obtained on the entire camera sensor due to low-coherence illumination, we can use the IDIA technique to create an interference area that is doubled in size compared to the initial low-coherence interference area. In the case 
that the off-axis interference is obtained on the entire camera sensor, we can use the IDIA technique to double the camera FOV. In any case, the FOV doubling is performed while sharing the camera dynamic range, and without compromising the imaging parameters, such as magnification and resolution. On the other hand, because narrowing the FOV of the digital camera can help speed up the camera frame rate, the technique also enables faster acquisition rates of highly dynamic objects or phenomena, while recording half of the original camera FOV and still being able to reconstruct the original FOV.

It should be noted that narrow camera FOV is also a problem with other types of imaging modalities, where FOV multiplexing can help increase the imaging system throughput. For example, high-resolution, wide-FOV imaging is a principal requirement in the fields of security and situational awareness. ${ }^{14}$ Several optical configurations have been suggested over the years ${ }^{15-18}$ to increase the recorded FOV without increasing the sensor size or losing resolution, but frequently with the sacrifice of time resolution or the extent of the dynamic areas allowed in the recorded image.

In contrast to these non-interferometric methods, and without losing time resolution, the IDIA principle proposes to multiplex two FOVs into a single off-axis interferogram to obtain simultaneous imaging with a wider FOV. Regular interferometry is known to be compressive when reconstructing a three-dimensional image from a single interferogram, ${ }^{19}$ but here we optically compress more interferometric information into a single multiplexed off-axis interferogram. Multiplexing several off-axis interferograms into a single interferogram and reconstructing the data encoded into each of them have already been shown to be feasible. ${ }^{20}$ This feature has been used in the past for other applications, which include solving the phase unwrapping problem, ${ }^{9,21}$ recording ultrafast events using suitable temporal encoding, $^{22-24}$ and obtaining super-resolution capabilities by synthetically increasing the numerical aperture (NA) of the imaging system. ${ }^{25-27}$

Although the IDIA principle can be implemented in various holographic and interferometric imaging systems, we implemented this technique by modifying the compact and portable interferometric module recently developed in our group, ${ }^{6,7}$ which can be connected directly to the digital camera to turn it into a powerful off-axis interferometric camera with a wider FOV.

\section{MATERIALS AND METHODS}

\section{The IDIA principle}

As shown in Figure 1a, the IDIA technique is based on optically multiplexing two FOVs of the sample and interfering them with a reference beam in off-axis geometry, such that the off-axis interferences from the two FOVs create straight parallel fringes with directions orthogonal to each other, and thus two parts of the image of the sample are recorded simultaneously on a single camera sensor. A possible optical experimental setup that implements this principle is described in the next section. In the case that the same reference beam is used for the two FOVs of the sample, the digital camera at the output of the interferometric system records three separable off-axis interferences: two between the reference beam and each of the sample beams and one
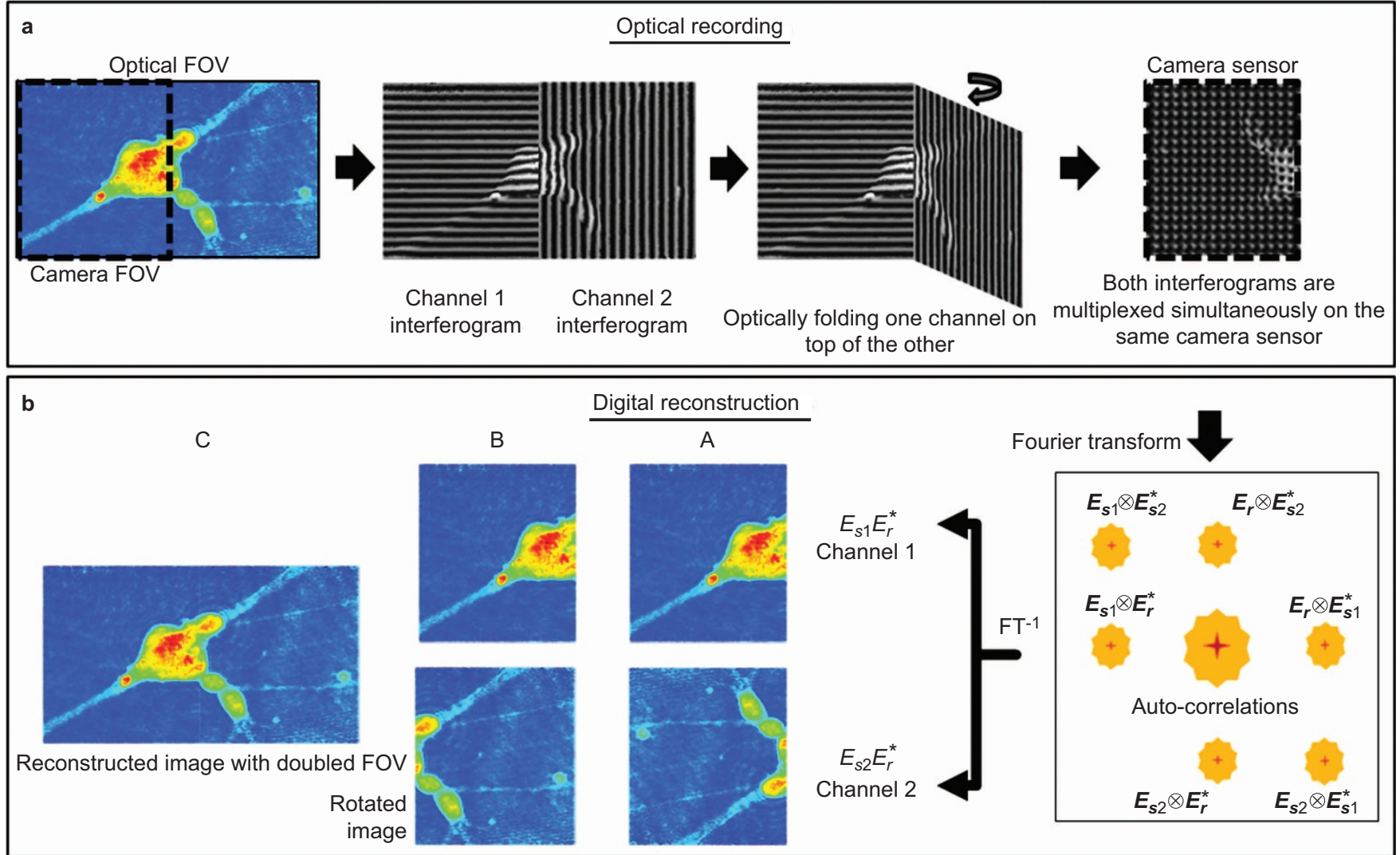

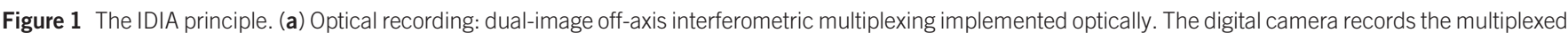

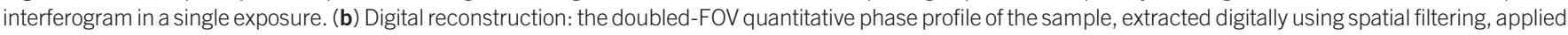

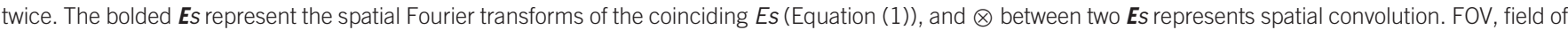
view; $\mathrm{FT}^{-1}$, inverse Fourier transform; IDIA, interferometry with doubled imaging area. 
between the two sample beams. Mathematically, this multiplexed interferogram can be described by the following equation:

$$
\begin{aligned}
\left|E_{s 1}+E_{s 2}+E_{r}\right|^{2}= & \left|E_{s 1}\right|^{2}+\left|E_{s 2}\right|^{2}+\left|E_{r}\right|^{2}+ \\
& E_{s 1} E_{s 2}^{*}+E_{s 2} E_{s 1}^{*}+E_{s 1} E_{r}^{*}+E_{r} E_{s 1}^{*}+E_{r} E_{s 2}^{*}+E_{s 2} E_{r}^{*}
\end{aligned}
$$

where $E_{s 1}$ and $E_{s 2}$ are the sample waves of two sample FOVs, and $E_{r}$ is the reference wave. According to the IDIA principle, by controlling the interference angle, the off-axis interference between $E_{s 1}$ and $E_{r}$ can be rendered orthogonal (rotated at $90^{\circ}$ ) to the off-axis interference between $E_{s 2}$ and $E_{r}$. As a result, the off-axis interference between $E_{s 1}$ and $E_{s 2}$ will be rotated at $45^{\circ}$ compared to any of the other two interference patterns. This $45^{\circ}$-rotated interference can be avoided by making sure that the two interferograms are created by orthogonally polarized light beams (not demonstrated in this paper).

In the spatial-frequency domain, the first three terms on the right side of Equation (1) are the auto-correlation elements, representing the intensities of the recorded waves. The other six terms on the right side of Equation (1) represent the cross-correlation elements between the waves. Due to the orthogonal off-axis interferences, and as illustrated in Figure 1b, these six cross-correlation elements are located on different areas of the Fourier plane. Therefore, two sample waves, corresponding to two sample FOVs, can be digitally extracted from the single multiplexed interferogram.

Regular off-axis interferometry, for capturing a single FOV, uses a single sample beam and a single reference beam, which results in two auto-correlation elements (resulting from terms 1 and 3 on the right side of Equation (1)) and two complex-conjugated cross-correlation elements (resulting from terms 6 and 7 on the right side of Equation (1)). Assuming that the maximum spatial frequency of the sample wave is $\omega_{c}$ (on each axis), each of the cross-correlation elements occupies a spatial bandwidth of $\left[-\omega_{c}, \omega_{c}\right]$, whereas the auto-correlation elements occupy a spatial bandwidth of $\left[-2 \omega_{c}, 2 \omega_{c}\right] .{ }^{28}$ In this case, to avoid an overlap between the cross-correlation elements and the autocorrelation elements, the center of the spatial-frequency contents of the cross-correlation elements is shifted to at least $\pm 3 \omega_{c}$ by adjusting the off-axis angle between the reference and sample beams, which requires a total spatial bandwidth of at least $8 \omega_{c}$. This encoding creates an empty space in the spatial-frequency domain, into which we insert the additional cross-correlation elements represented in Equation (1), and thus, FOV multiplexing is possible.

The reconstruction procedure includes an off-axis interferometric digital process (described in detail in other publications 1, 5, 7), applied twice. In the first time, it includes the spatial filtering of $E_{s 1} E_{r}^{*}$, and in the second time, it includes the spatial filtering of $E_{s 2} E_{r}^{*}$. The resulting phase profiles are then unwrapped digitally to avoid $2 \pi$ ambiguities. The two FOVs of the sample can be chosen to be located in adjacent places on the sample, so that after the reconstruction process a single continuous and double-sized FOV of the sample is obtained, as illustrated in Figure 1b.

\section{Experimental setup for implementing the IDIA principle}

A possible optical setup for implementing the IDIA principle described above is presented in Figure 2. The IDIA module, composed of a dual-image off-axis $\tau$ interferometer, ${ }^{7}$ is a portable interferometric module connected to the digital camera located at the output of the imaging system. The image from the coherently or partially coherently illuminated object is projected onto the input of the module instead of directly onto the digital camera. In the interferometric module, the image is optically Fourier transformed by lens L1, while being split into two beams using beam splitter

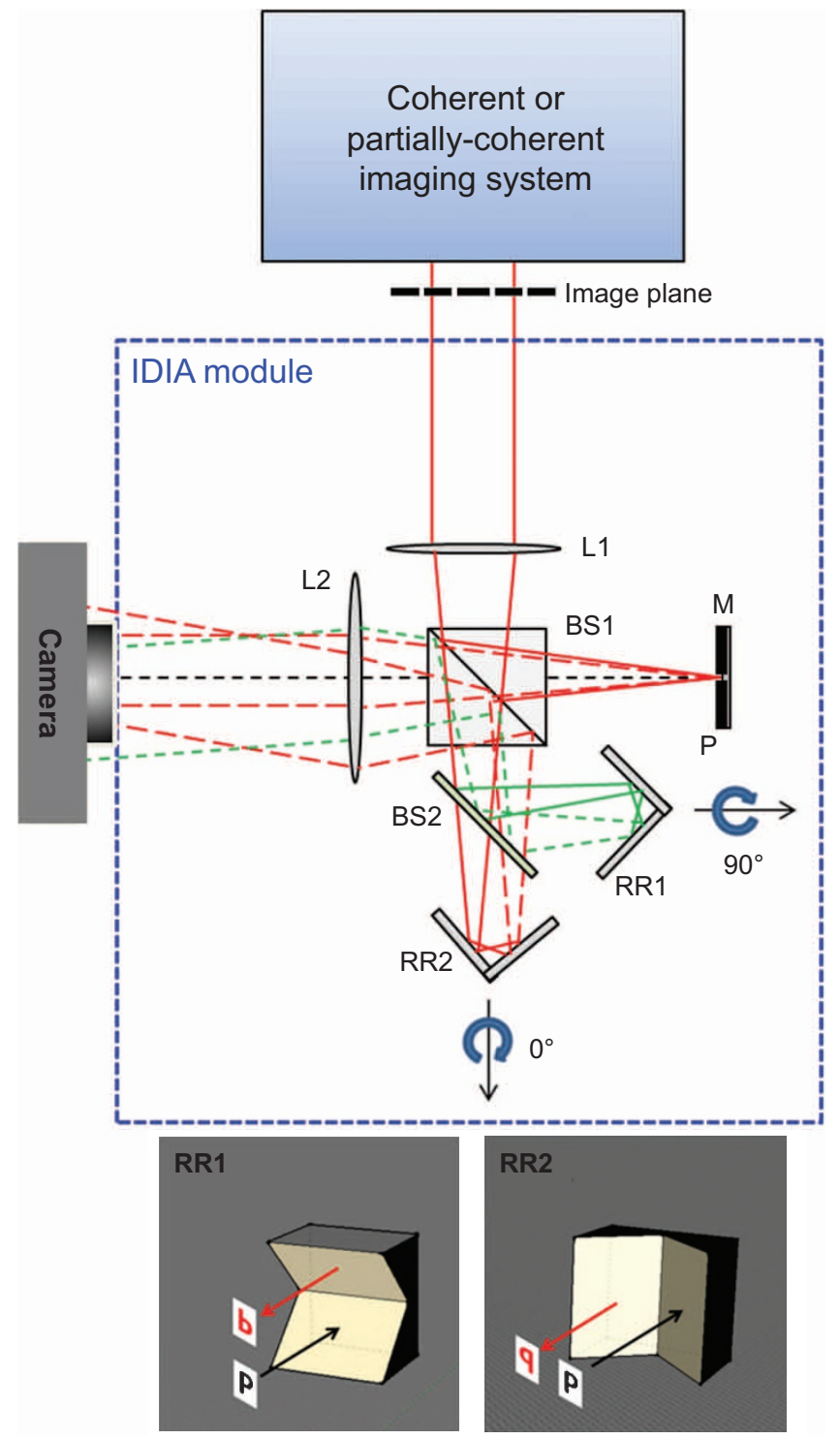

Figure 2 The IDIA module (dual-image off-axis $\tau$ interferometer) connected to the digital camera at the output of a coherent or partially coherent imaging system. The retro-reflectors are orthogonal to each other. Bottom inset: three-dimensional diagram of the retro-reflectors and their effect on the incoming image. For a three-dimensional diagram of the entire system, see Supplementary Fig. S1. BS, beam splitter; IDIA, interferometry with doubled imaging area; L1 and L2, lenses in a $4 \mathrm{f}$ configuration; $\mathrm{M}$, mirror; $\mathrm{P}$, pinhole; RR1 and RR2, retro-reflectors, each built out of two mirrors connected at a right angle.

BS1. One of the beams is filtered by pinhole $\mathrm{P}$ and reflected by mirror $\mathrm{M}$, where the pinhole transfers only a very small bandwidth around the zero spatial frequency, and thus creates a reference beam by effectively erasing the sample information. ${ }^{6,7}$ Next, the reference beam is optically Fourier transformed back to the camera plane by lens L2, where lenses L1 and L2 are positioned in a $4 \mathrm{f}$ lens configuration (i.e., the distance between the image plane in the output of the imaging system and L1 is equal to the lens focal length, the distance between L1 and L2 is equal to sum of their focal lengths, and the distance between lens L2 and the camera sensor is equal to the focal length of L2). The second beam that propagates from BS1 is split again by beam splitter BS2, and both sample beams are reflected by two retro-reflectors, RR1 and RR2, 
and optically Fourier transformed back to the camera plane by lens L2. Each of the retro-reflectors is used to shift the actual Fourier-plane center. The two retro-reflectors are positioned perpendicular to each other, so that one creates a Fourier-plane shift in the $x$ direction and the other one creates a Fourier-plane shift in the $y$ direction. The retro-reflector three-dimensional positions are illustrated in the bottom of Figure 2 and in Supplementary Fig. S1. This arrangement creates multiplexing of two linear, perpendicular off-axis interference fringe patterns that can be recorded simultaneously by a single camera exposure. Because the retro-reflectors also rotate the image of the sample, we obtain two mirrored images on the camera. According to this arrangement, there is an area where the two overlapping images from the two retro-reflectors are continuous. In this area, we can reconstruct two different complex wavefronts originating from the two continuous FOVs of the sample. Because these wavefronts are mirrored, one of them needs to be digitally rotated (see step B, bottom channel in Figure 1b) and stitched to the first one to create an image with a doubled FOV (see step C in Figure 1b).

When using a low-coherence source, the off-axis interference area is typically limited by the coherence length of the source. ${ }^{9}$ This area might be smaller than the camera sensor size. However, in our case, because the dual-image off-axis $\tau$ interferometer records two independent interferences, each channel will have its own interference area limited by the coherence length of the source. For this reason, it is possible to double the interferometric FOV using the IDIA principle even if using a low-coherence source that limits the interferometric FOV, provided that the camera sensor is square, whereas for other camera sensor geometries, significant interferometric FOV extension, which is close to doubling, is possible. Figure 3 illustrates the relations among the optical FOV, the camera FOV, and the coherence-limited interferometric FOV for the cases of high-coherence and low-coherence illumination sources.

\section{RESULTS AND DISCUSSION}

Interferometric phase microscopy setup and initial tests

Using the proposed technique, we demonstrate a significant increase in the interferometric FOV for IPM, which is one of the most rapidly growing interferometric fields in the last decade. For these demonstrations, we used the inverted transmission microscope shown in Figure 4, containing three different infinity-corrected microscope objectives, one with a $10 \times$ magnification and a $0.25 \mathrm{NA}$, the second with a $40 \times$ magnification and a $0.66 \mathrm{NA}$, and the third with a $63 \times$ magnification and a 1.4 NA (oil immersion). The IDIA module described in the previous section was connected at the camera port of the microscope, and a digital camera (DCC1545M, monochromatic CMOS camera with $1280 \times 1024$ square pixels of $5.2 \mu \mathrm{m}$; Thorlabs, Newton, NJ, USA) was connected to the output of the module. The diameter of pinhole $\mathrm{P}$ was $30 \mu \mathrm{m}$, and lenses $\mathrm{L} 1$ and $\mathrm{L} 2$ were achromatic lenses with focal lengths of $100 \mathrm{~mm}$ and $125 \mathrm{~mm}$, respectively, creating total magnifications of $12 \times, 47 \times$ and $74 \times$, respectively. The transmission microscope was illuminated by a low-coherence light source with a central wavelength of $510 \mathrm{~nm}$ and a full-width-at-half-maximum bandwidth of $6.1 \mathrm{~nm}$, creating a coherence length of $13.58 \mu \mathrm{m}$. This light source is composed of a supercontinuum fiber-laser source (SC4004; Fianium, Southampton, UK) connected to a computer-controlled acousto-optical tunable filter (Fianium). In addition, to demonstrate the utility of the method for doubling the entire camera FOV for highly coherent sources, we alternatively integrated a HeNe laser (with $632.8 \mathrm{~nm}$ wavelength) as the illumination source of the microscope.

Using the low-coherence source (which limits the interferometric FOV) without the IDIA technique, we obtained off-axis interference on $1280 \times 800$ pixels of the camera, on a total area of $6.66 \mathrm{~mm} \times 4.16 \mathrm{~mm}=27.7 \mathrm{~mm}^{2}$. When using the IDIA technique, which multiplexes the two interferometric FOVs, the off-axis interference area on the camera sensor was $4.16 \mathrm{~mm} \times 5.32 \mathrm{~mm}(800 \times 1024$

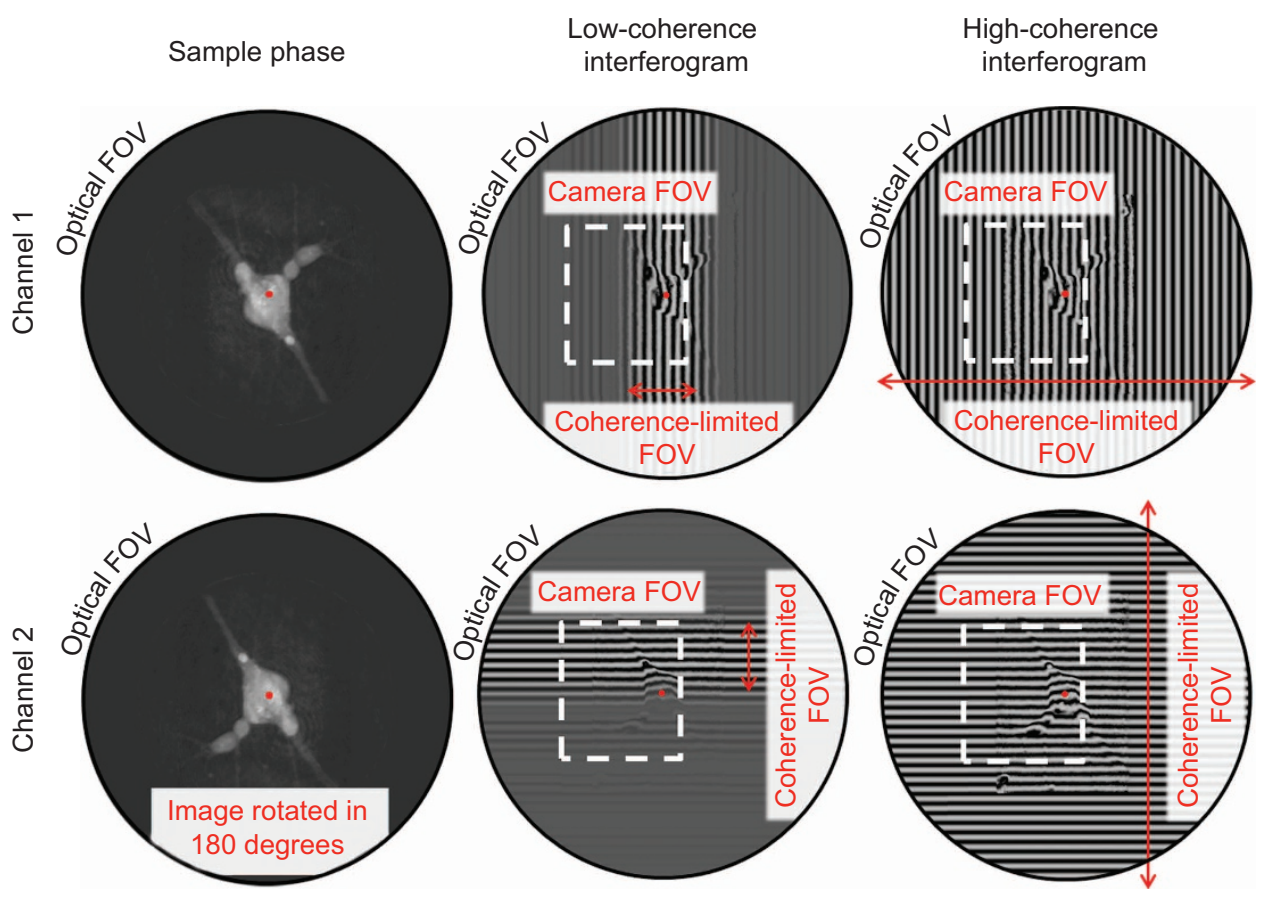

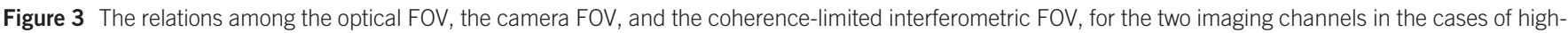
coherence illumination and low-coherence illumination. The red dot indicates the center of the optical FOV. FOV, field of view. 


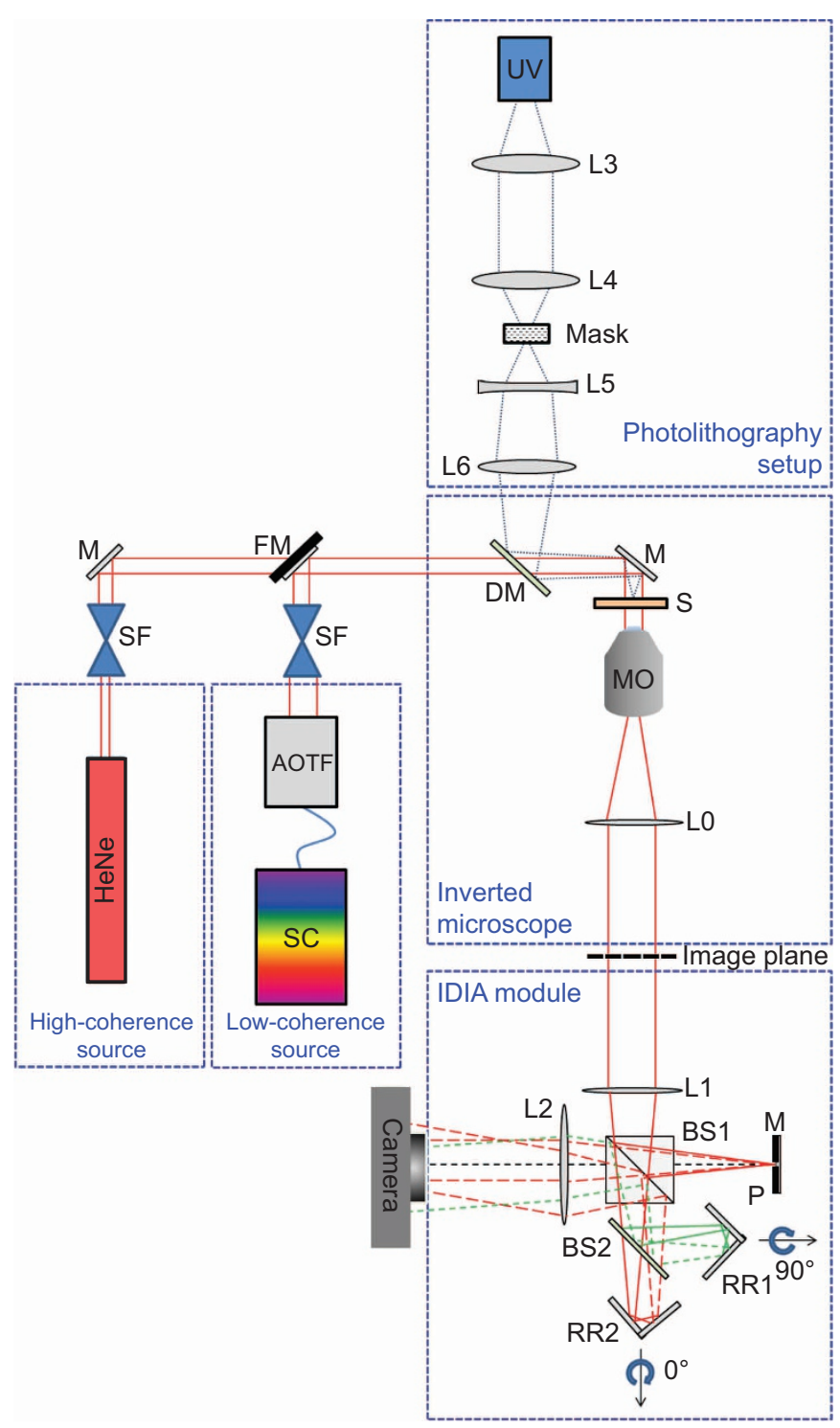

Figure 4 Experimental setup for demonstrating the IDIA principle: an inverted transmission microscope, where the IDIA module is connected to its output. For some of the experiments, we used low-coherence illumination, whereas for the rest of the experiments, we used high-coherence illumination. For the last experiment, we used the photolithography setup integrated into the microscope. AOTF, acousto-optical tunable filter; BS1 and BS2, beam splitters; DM, dichroic mirror; FM, flip mirror (for light-source selection); HeNe, helium-neon laser; IDIA, interferometry with doubled imaging area; LO, L1, L2, L3, L4, L5 and L6, lenses; M, mirror; Mask, photolithography mask; MO, microscope objective; RR1 and RR2, retro-reflectors; S, sample; SC, supercontinuum light source; SF, spatial filter (two lenses and a confocally positioned pinhole); UV, ultraviolet light-emitting diode.

pixels) for the first channel and $4.16 \mathrm{~mm} \times 6.66 \mathrm{~mm}(800 \times 1280$ pixels $)$ for the second channel.

To evaluate the system sensitivity, we first recorded 240 sample-free interferograms during 12 seconds, and for each of them, we calculated the optical thickness profile, proportional to the quantitative phase profile. We obtained average temporal optical thickness stability levels of $0.71 \mathrm{~nm}$ and $0.63 \mathrm{~nm}$ for the first and second channels, respectively, and average spatial optical thickness stability levels of $0.88 \mathrm{~nm}$ and $0.91 \mathrm{~nm}$ for the first and second channels, respectively.

In the specific demonstrations described below, we recorded interferograms of samples that are too large to fit into a single interferometric
FOV, as defined by the coherence length of the low-coherence source mentioned above. In these demonstrations, we allowed a $12 \%$ overlap between the channels to facilitate the stitching of the final images. Therefore, the total recorded interference area, after stitching the two channels, was $47.07 \mathrm{~mm}^{2}$, which was $70 \%$ larger than the original recorded interference area, and even larger than the camera sensor size $\left(35.44 \mathrm{~mm}^{2}\right)$. Without this $12 \%$ overlap, we were able to increase the total recorded area to $49.84 \mathrm{~mm}^{2}$, which is $80 \%$ larger than the original interference area. Note that in this demonstration, because the camera sensor is not square, we cannot record the same interference areas from both channels, so a full 100\% increase in the recorded offaxis interference area is not possible for non-square camera sensors.

The initial test included low-coherence interferometric imaging of the thickness profile of groups 8-11, elements 1-6 (line width of 1.95$0.135 \mu \mathrm{m}$ ) of an optically transparent 1951 USAF test target, which we created by focused-ion-beam lithography on glass. Figure 5a shows the recorded multiplexed interferogram, using the $40 \times$ objective (defining optical resolution limit of $604.6 \mathrm{~nm}$ ), and Figure 5b shows the absolute value of its spatial Fourier transform, presenting the various well-separated elements represented in Equation (1) and on the bottom-right side of Figure 1b. From this spatial-frequency plane, we digitally filtered the cross-correlation elements marked by the white boxes to yield the quantitative optical thickness maps of the two FOVs. Figure $5 c$ shows the final optical thickness maps from both channels, stitched together. As shown in this image, we can discriminate the various test-target details down to group 9, element $5(620 \mathrm{~nm})$, corresponding to the optical-system resolution limit.

To confirm that it is not possible to extend the FOV and keep the same resolution limit by simply decreasing the magnification, we measured the same test target with a magnification reduced by a factor of 2 . This measurement was performed by adding a $4 \mathrm{f}$ lens imaging system after the microscope with a magnification of 0.5 , while retaining the optical resolution limit. For a valid comparison, in both cases, the filtering window (marked by a white box in Figure 5) size was equal to $2 \omega_{c}$ (resulting from the microscope optical-resolution limit). Figure $5 \mathrm{~d}$ shows the recorded regular off-axis interferogram, and Figure 5 e shows the corresponding spatial-frequency plane, illustrating the overlap between the auto-correlation and the cross-correlation elements resulting from the demagnification. Figure $5 \mathrm{f}$ shows the reconstructed optical-thickness profile obtained by filtering the cross-correlation element marked by a white box in Figure 5e, demonstrating a resolution limit of $0.77 \mu \mathrm{m}$ (group 9, element 3). Therefore, if extending the recorded FOV by simply demagnifying, resolution loss occurs in the resulting image due to the overlap between the auto-correlation and the cross-correlation elements, in addition to significant reduction of the image quality. It is possible to optimize the image quality of Figure $5 \mathrm{f}$ by narrowing the spatial filtering window. However, the resolution limit in this case further decreases due to filtering of high spatial frequencies. For example, in Supplementary Fig. S2, we filtered the cross-correlation by a half-wide window, resulting in a clearer image but with a resolution limit of only $1.1 \mu \mathrm{m}$ (group 8, element 5).

\section{Quantitative imaging of biological cells}

We next used the IDIA technique to extend the FOV in low-coherence interferometric imaging of the quantitative optical thickness profile of embryonic rat brain specimens containing both neurons and glial cells, using the $40 \times$ objective. As shown in Figure 6, these cells, despite their relatively small size, have long extensions including micronscale-diameter axons between the neurons in the network, and thus, 


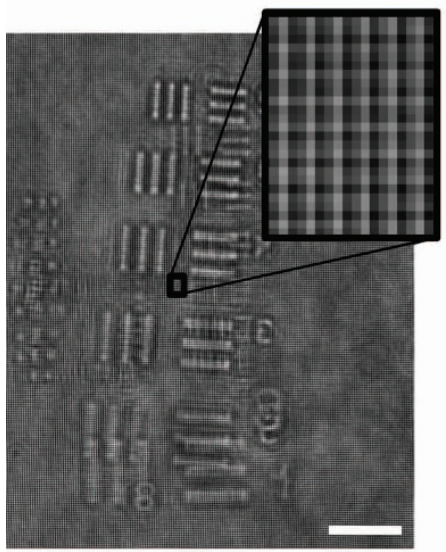

a

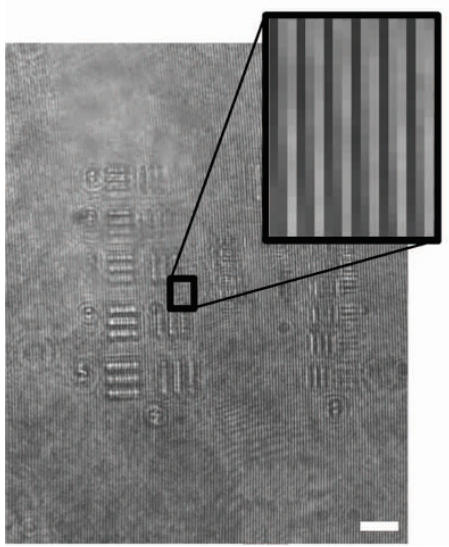

d

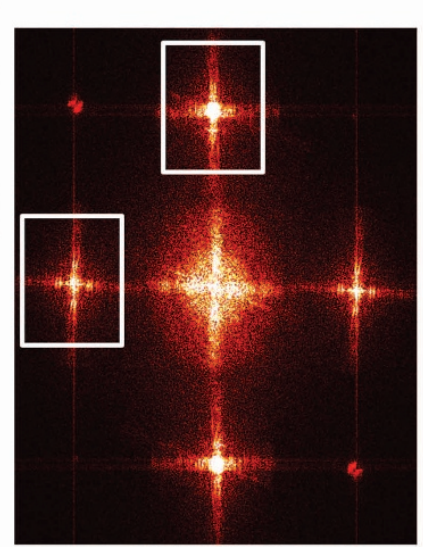

b

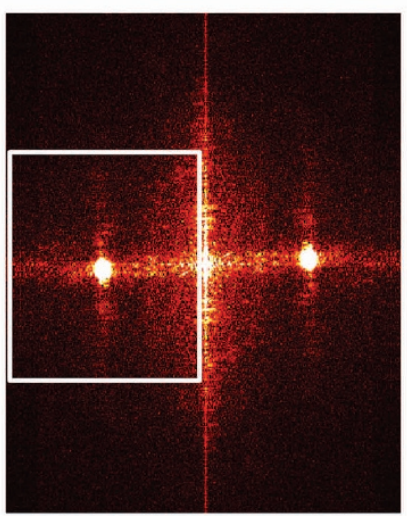

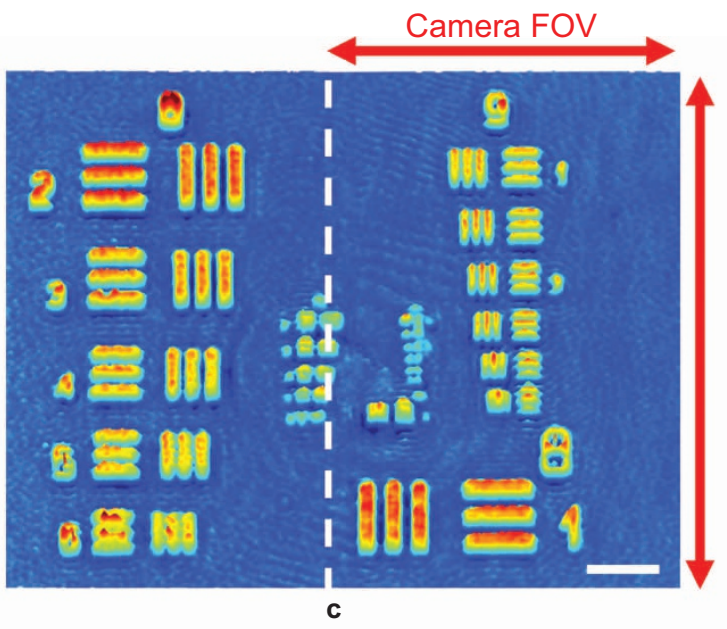

Optical thickness

(nm)

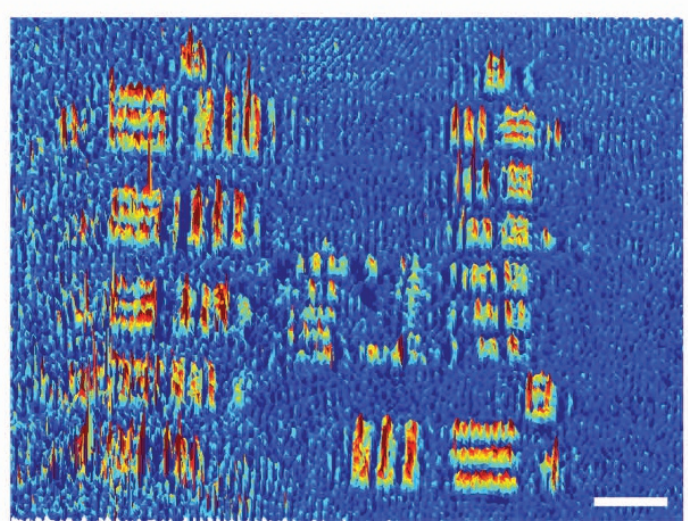

f

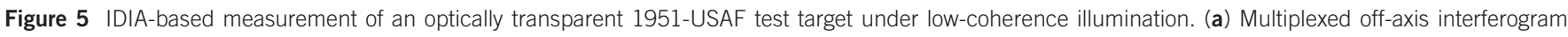

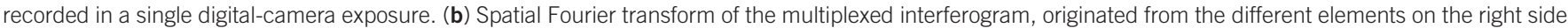

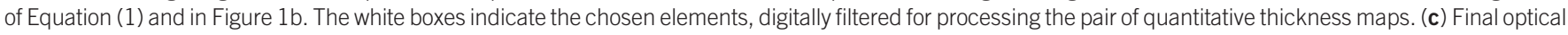

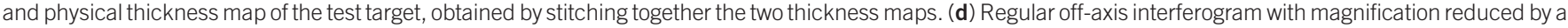

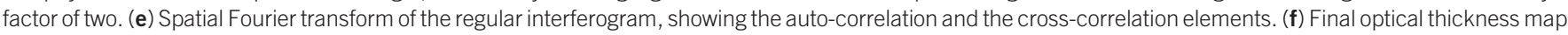

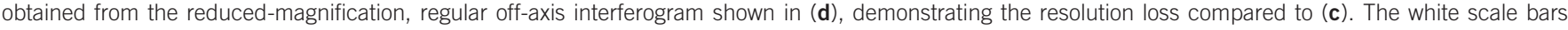
represent $10 \mu \mathrm{m}$. An additional spatial filtering option is shown in Supplementary Fig. S2. FOV, field of view; IDIA, interferometry with doubled imaging area.

high magnification is needed to image them in detail. The stitched optical thickness map of this nerve network obtained using the IDIA technique is shown in Figure 6a. This optical thickness map cannot be recorded in a single exposure by regular off-axis interferometric setups with the specific camera and magnification used, as a single off-axis interferometric FOV would not be wide enough to contain the entire detailed image. Another example of an IDIA-based optical thickness map of brain cells is shown in Figure 6b. Again, although the image of this sample is larger than the off-axis interferometric FOV, we have successfully multiplexed two interferometric FOVs and recorded them in a single camera exposure.

Because the camera FOV and frame rate are interchangeable, the IDIA principle can also be used to increase dramatically the camera frame rate, while recording half of the FOV and still reconstructing the original camera FOV. To demonstrate this ability, we imaged a human sperm cell in motion. A human spermatozoon has a small flat oval head, with 3-5 $\mu \mathrm{m}$ diameter and $1 \mu \mathrm{m}$ thickness. The spermatozoon tail is $50-60 \mu \mathrm{m}$ long (including the neck connecting the tail to the head) and $200-400 \mathrm{~nm}$ in diameter. ${ }^{29}$ Interferometry has been shown to be useful for various sperm tests. ${ }^{30-32}$ However, to quantitatively record a single spermatozoon with its finely detailed tail using a high magnification and high-NA microscope objective, a small pixel size camera must be used. For a given camera, these restrictions might create an optical FOV that is larger than the camera FOV, which reduces the user's ability to record the long tail and the relatively large head together in a single camera exposure.

In addition, the spermatozoon has a complex motion; its tail is characterized by three-dimensional helix rotation and its head has three-axial rotation. $^{29}$ Furthermore, the entire spermatozoon moves rapidly at speeds ranging from 48 to $162 \mu \mathrm{m} \mathrm{s}^{-1,33}$ making it hard to scan its image during movement. In this case, the IDIA principle can allow us to quantitatively record structural spermatozoon anomalies such as double-tailed or double-headed morphologies, whereas the scan-free nature of this wide-field, doubled-FOV quantitative imaging technique allows us to image its fast and complex dynamics.

To be able to image an entire human spermatozoon at 80 frames per second using the $63 \times$ objective, one would need a camera 


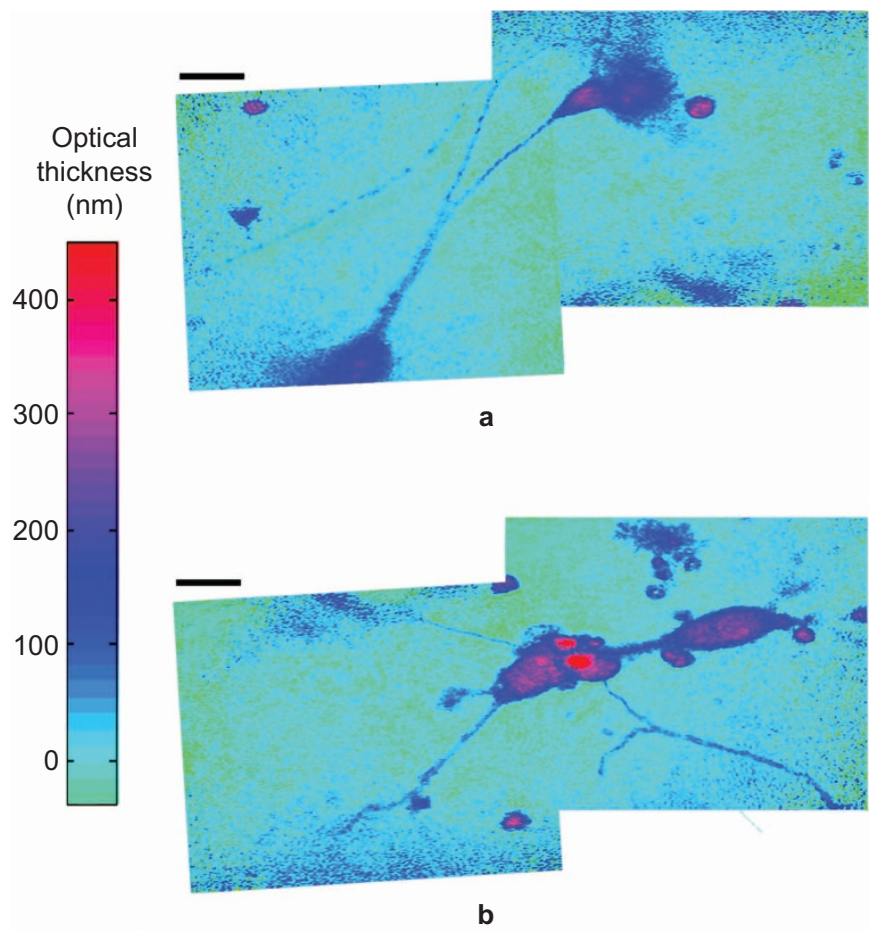

Figure 6 Quantitative optical thickness maps of an embryonic rat brain specimen, each of which is obtained by the IDIA technique in a single camera exposure, enabling the recording of a wider FOV with the fine neuronal details. (a) Network of two nerves connected to an unspread glial cell. (b) Neural sample containing both nerves and glial cells. The color bar shows the quantitative optical thickness of the sample in nanometers. The black scale bars represent $10 \mu \mathrm{m}$. FOV, field of view; IDIA, interferometry with doubled imaging area.

sensor size of $3.7 \mathrm{~mm} \times 2.7 \mathrm{~mm}$. With the camera used in our experiment, containing a pixel size of $5.2 \mu \mathrm{m}$ square, this sensor-size requirement translates to $710 \times 512$ pixels. However, this specific camera can record 80 frames per second for a FOV of $512 \times 512$ pixels, which is not enough to image the entire spermatozoon in motion. However, by implementing the IDIA technique (with an overlapping area of $8 \%$ ), we are able to increase the recorded FOV from $512 \times 512$ pixels to $512 \times 980$ pixels, while still enabling acquisition rate of 80 frames per second, which allows us to see the entire spermatozoon motion.

Figure 7 shows the motion of the spermatozoon in space at four different time points, and Supplementary Movie 1 shows its entire dynamics, where it is possible to see the complex three-dimensional movement of the spermatozoon, including its head rotation and the helical movement of the tail. As shown in Figure 7b, even when the cell head has passed completely to the second part of the frame, in the first part of the frame the entire tail is observed, demonstrating the need for the IDIA technique to provide wider FOV in this case. In the bottom right of Supplementary Movie 1, it is possible to see the dynamic multiplexed interferogram being directly recorded by the digital camera (before processing it into the optical-thickness profile shown on the left). As can be observed in this dynamic multiplexed interferogram, the spermatozoon appears in two places at once due to the optical folding of the two FOVs and the small overlap between them. Also observable in Supplementary Movie 1 is the rapid flow of the medium around the spermatozoon, which, in spite of being very fast, is continuous between the two FOVs.

The ability to quantitatively image the entire detailed movement of a single spermatozoon can be important in choosing a spermatozoon for in vitro fertilization, as it is expected to provide a better indication of the cell health based on both the cell detailed structure and its dynamics.

Of course, other larger, faster (and probably more expensive) cameras exist, but even for them, the IDIA principle can provide doubled FOV or, alternatively, faster frame rate.

\section{Real-time non-destructive profilometry during lithography processes}

Our next demonstration includes quantitative thickness imaging during the rapid generation of thin elements by projection photolithography. The ability to record and test the quality of larger FOVs is important for
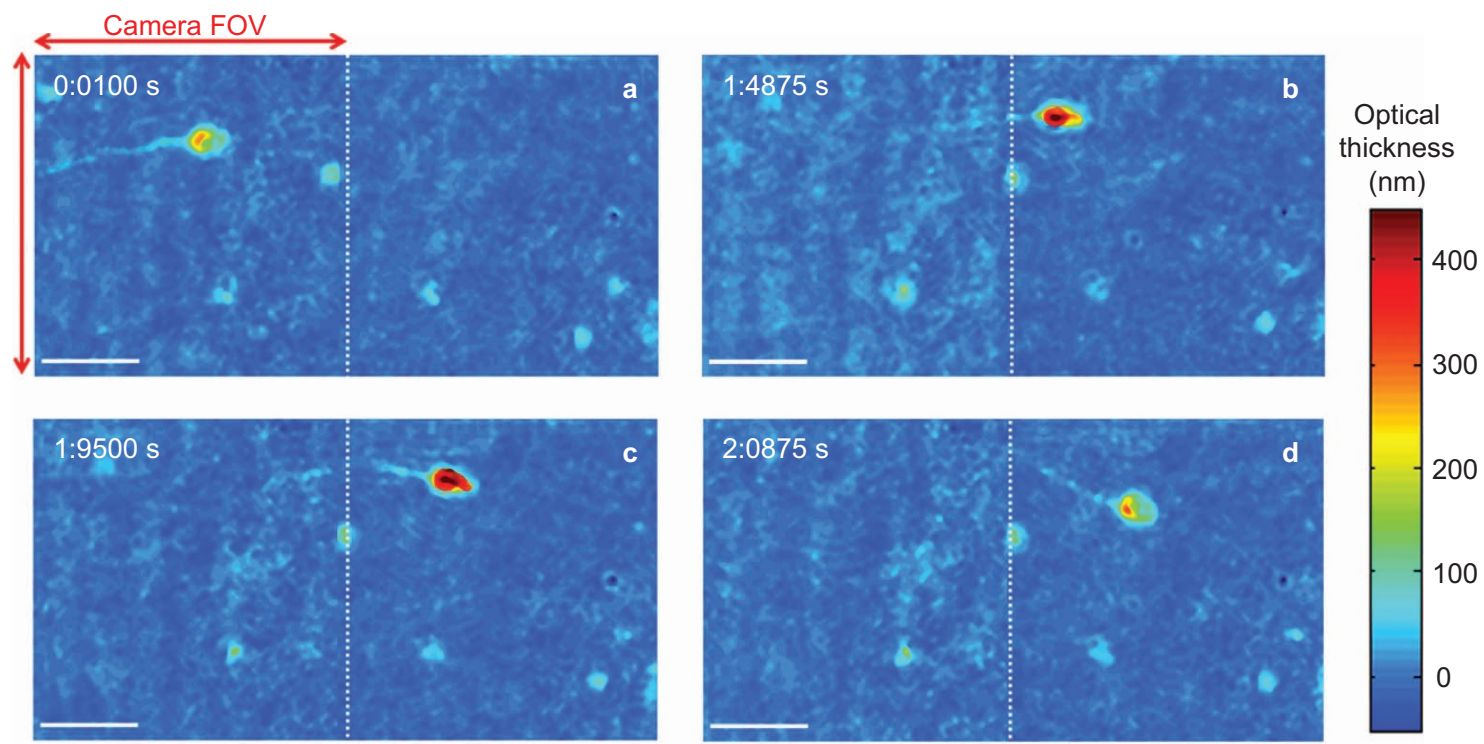

Figure 7 Quantitative optical thickness maps of a human spermatozoon swimming in 50\% semen and $50 \%$ phosphate-buffered saline, as recorded by the IDIA technique, enabling the acquisition of the fast dynamics of the spermatozoon with fine details on a doubled FOV. The white dashed line indicates the location of the stitching between the two FOVs. The color bar represents the quantitative optical thickness of the sample in nanometers. The white scale bars represent $10 \mu \mathrm{m}$. See the dynamics in Supplementary Movie 1. FOV, field of view; IDIA, interferometry with doubled imaging area. 


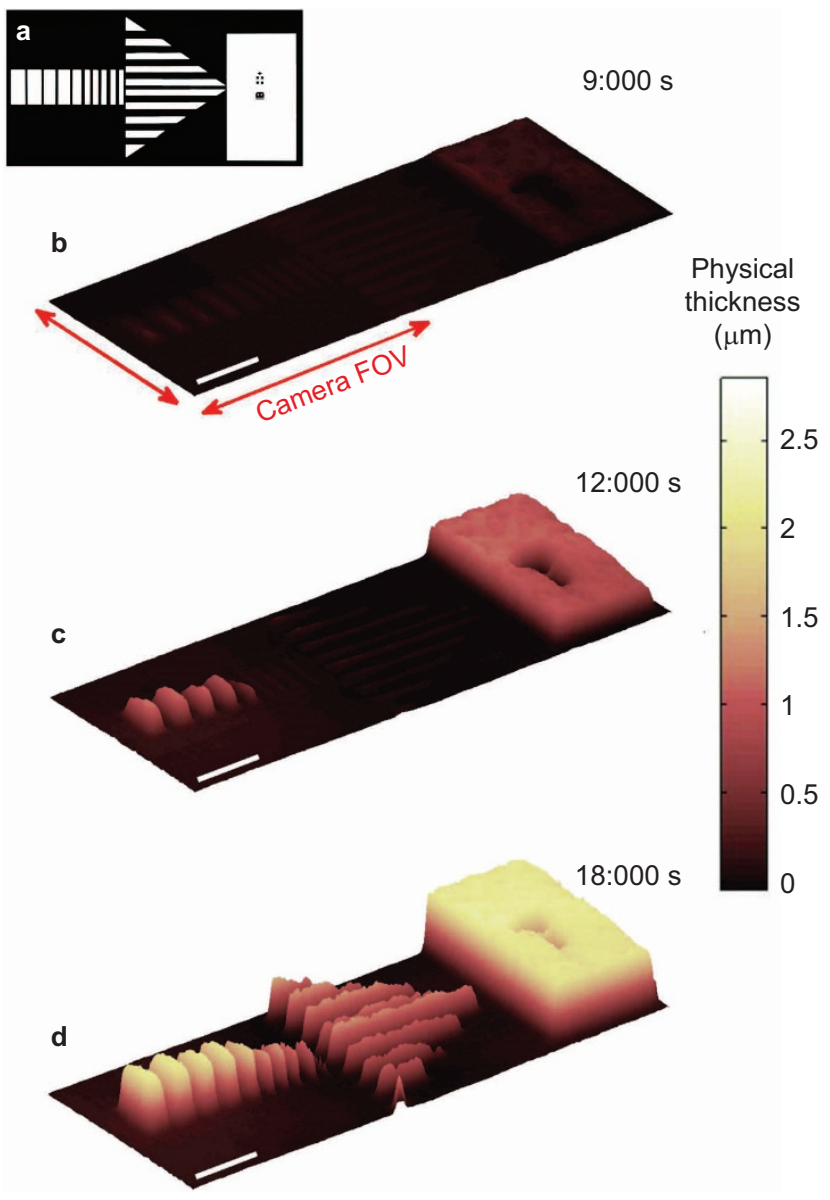

Figure 8 Quantitative thickness imaging of a rapid lithography process with a doubled camera FOV, as obtained by the IDIA technique. (a) The photolithography mask used. (b-d) The physical thickness profile of the element at different time points during the curing of the photoresist in the lithography process. The color bar represents the quantitative physical thickness of the sample in micrometers. The white scale bars represent $150 \mu \mathrm{m}$. See the dynamics in Supplementary Movie 2. FOV, field of view; IDIA, interferometry with doubled imaging area.

mass production during the creation of lithographed elements, such as during the fabrication of integrated circuits, ${ }^{34}$ as a larger number of elements or wider objects could be produced and examined at a given time. For this demonstration, we integrated an additional lithography imaging system into the microscope, so that we can rapidly image the lithographed element during the curing of photoresist adhesive (NOA 81). This measurement can be used to control the curing depths and structures and to provide feedback to the lithography system in real time. A scheme of this additional photolithography setup is shown in the upper part of Figure 4 . This setup included a $0.1 \times$-magnification optics cable of imaging the mask onto the sample plane $S$ for projection photolithography. For this experiment, we used the mask shown in Figure 8a. A $375 \mathrm{~nm}, 0.27 \mathrm{~W} \mathrm{~cm}^{-2}$ light-emitting diode was used to illuminate the mask under Köhler illumination. A $490 \mathrm{~nm}$ cutoff filter was placed before the tube lens in the infinity space to prevent the diode light from reaching the detector (the filter is not shown in the figure). Then, using the transmission microscope described above, integrated with the IDIA module, we imaged the quantitative optical thickness profile of the lithographed element during the curing process, under $10 \times$ magnification. In this experiment, the highly coherent source was used in the input of the microscope, creating interference across the entire camera sensor, and therefore, using IDIA, we were able to effectively record a doubled camera FOV.

Figure $8 \mathrm{~b}-8 \mathrm{~d}$ and Supplementary Movie 2 present the optical thickness profile of the element during the curing of the photoresist at different time points. The structure and the refractive index of the polymer change during curing, whereas the physical thickness of the photoresist remains constant.

Note that this lithographed element could not be imaged by the given camera under the chosen magnification in a single camera exposure in an off-axis interferometric manner, because the projected image is larger than the camera sensor size. Here as well, scanning this dynamic element might result in a loss of information. However, by implementing the IDIA principle, we were able to fully record this element during the adhesive curing, which provides the opportunity to control and test the lithography process.

\section{CONCLUSIONS}

We have presented the IDIA technique, which enables doubling the off-axis interferometric FOV. Using this principle, we partially solve the problem of the narrow interference area associated with off-axis interferometric setups integrated with low-coherence light sources. As we multiplex two interferometric channels in a single camera FOV, we can reconstruct a single extended image, which is equal in size to twice the off-axis interferometric FOV that can be conventionally recorded by the camera in a single exposure. On the other hand, when using highly coherent illumination, allowing us to obtain interference on the entire camera sensor, we double the FOV of the entire camera sensor regardless of the initial interference area. Hence, in the case that the interferometric FOV is smaller than the camera sensor, we can double the interferometric FOV. In the case that the interferometric FOV is larger than the camera sensor, we can double the FOV defined by the size of entire camera sensor.

Of course, because the camera FOV and acquisition frame rate are interchangeable, the IDIA technique encapsulates the capability of increasing the camera frame rate without decreasing the original reconstructed FOV, as instead of doubling the camera FOV, the acquisition frame rate can be increased, while using half of the camera pixels and still reconstructing the original FOV.

Our demonstrations included detailed off-axis interferometric phase imaging of brain cell samples, a rapid movement of a sperm cell, and a rapid photolithography process. Although we have implemented the IDIA principle by modifying our unique, compact and portable $\tau$ interferometer, the principle of IDIA presented in this paper can be integrated into other holographic and interferometric imaging setups, providing wider FOVs or faster acquisition rates for a given camera, and thus enabling the imaging of larger and rapidly changing samples.

Finally, as each channel contains not only the quantitative phase map, but also the intensity profile of the sample, one can also use the IDIA technique in reflection mode on non-transparent, reflective samples to create panoramic images and gain extended FOVs.

\section{ACKNOWLEDGMENTS}

This work is supported by the FP7 Marie Curie Career Integration Grant.

1 Girshovitz P, Shaked NT. Generalized cell morphological parameters based on interferometric phase microscopy and their application to cell life cycle characterization. Biomed Opt Exp 2012; 3: 1757-1773.

2 Marquet P, Rappaz B, Magistretti PJ, Cuche E, Emery Y et al. Digital holographic microscopy: a noninvasive contrast imaging technique allowing quantitative 
visualization of living cells with subwavelength axial accuracy. Opt Lett 2005; 30 468-470.

3 Kemper B, Bauwens A, Vollmer A, Ketelhut S, Langehanenberg P et al. Label-free quantitative cell division monitoring of endothelial cells by digital holographic microscopy. J Biomed Opt 2010; 15: 036009.

4 Popescu G, Ikeda T, Dasari RR, Feld MS. Diffraction phase microscopy for quantifying cell structure and dynamics. Opt Lett 2006; 31: 775-777.

5 Qu WJ, Choo CO, Yu YJ, Asundi A. Microlens characterization by digital holographic microscopy with physical spherical phase compensation. App/ Opt 2010; 49: 64486454.

6 Shaked NT. Quantitative phase microscopy of biological samples using a portable interferometer. Opt Lett 2012; 37: 2016-2018.

7 Girshovitz P, Shaked NT. Compact and portable low-coherence interferometer with off-axis geometry for quantitative phase microscopy and nanoscopy. Opt Exp 2013; 21: 5701-5714.

8 Martínez-León L, Pedrini G, Osten W. Applications of short-coherence digital holography in microscopy. Appl Opt 2005; 44: 3977-3984.

9 Monemhaghdoust Z, Montfort F, Emery Y, Depeursinge C, Moser C. Dual wavelength full field imaging in low coherence digital holographic microscopy. Opt Exp 2011; 19: 24005-24022.

10 Kolman P, Chmelík R. Coherence-controlled holographic microscope. Opt Exp 2010; 18: 21990-22003.

11 Ansari Z, Gu Y, Tziraki M, Jones R, French PM. Elimination of beam walk-off in lowcoherence off-axis photorefractive holography. Opt Lett 2001; 26: 334-336.

12 Leith EN, Swanson GJ. Achromatic interferometers for white light optical processing and holography. Appl Opt 1980; 19: 638-644.

13 Maznev AA, Crimmins TF, Nelson KA. How to make femtosecond pulses overlap. Opt Lett 1998; 23: 1378-1380.

14 Uttam S, Goodman NA, Neifeld MA, Kim C, John R et al. Optically multiplexed imaging with superposition space tracking. Opt Exp 2009; 17: 1691-1713.

15 Marcia RF, Kim C, Eldeniz C, Kim J, Brady DJ et al. Superimposed video disambiguation for increased field of view. Opt Exp 2008; 16: 16352-16363.

16 Decker JA, Harwitt MO. Sequential encoding with multislit spectrometers. Appl Opt 1968; 7: 2205-2209.

17 Decker JA. Experimental realization of the multiplex advantage with a Hadamard transform spectrometer. Appl Opt 1971; 10: 510-514.

18 Gehm ME, McCain ST, Pitsianis NP, Brady DJ, Potuluri P et al. Static two-dimensional aperture coding for multimodal, multiplex spectroscopy. Appl Opt 2006; 45: 2965 2974.

19 Brady DJ, Choi K, Marks DL, Horisak R, Lim S. Compressive holography. Opt Exp 2009; 17: 13040-13049.
20 Paturzo M, Memmolo $\mathrm{P}$, Tulino A, Finizio A, Ferraro $\mathrm{P}$. Investigation of angular multiplexing and de-multiplexing of digital holograms recorded in microscope configuration. Opt Exp 2009; 17: 8709-8718.

21 Ferraro P, Miccio L, Grilli S, Paturzo M, de Nicola S et al. Quantitative phase microscopy of microstructures with extended measurement range and correction of chromatic aberrations by multiwavelength digital holography. Opt Exp 2007; 15: 14591-14600.

22 Liu Z, Centurion M, Panotopoulos G, Hong J, Psaltis D. Holographic recording of fast events on a CCD camera. Opt Lett 2002; 27: 22-24.

23 Wang $X$, Zhai $\mathrm{H}, \mathrm{Mu}$ G. Pulsed digital holography system recording ultrafast process of the femtosecond order. Opt Lett 2006; 31: 1636-1638.

24 Gabolde P, Trebino R. Single-shot measurement of the full spatio-temporal field of ultrashort pulses with multi-spectral digital holography. Opt Exp 2006; 14: 11460-11467.

25 Liu C, Liu Z, Bo F, Wang Y, Zhu J. Super-resolution digital holographic imaging method. Appl Phys Lett 2002; 81: 3143-3145.

26 Paturzo M, Merola F, Grilli S, De Nicola S, Finizio A et al. Super-resolution in digital holography by a two-dimensional dynamic phase grating. Opt Exp 2008; 16: 17107 17118.

27 Micó V, Zalevsky Z, Ferreira C, García J. Superresolution digital holographic microscopy for three-dimensional samples. Opt Exp 2008; 16: 19260-19270.

28 Goodman JW. Introduction to Fourier Optics. New York: McGraw-Hill; 1996. pp 295392.

29 Smith DJ, Gaffney EA, Blake JR, Kirkman-Brown JC. Human sperm accumulation near surfaces: a simulation study. J Fluid Mech 2009; 621: 289-320.

30 Su TW, Choi I, Feng J, Huang K, McLeod E et al. Sperm trajectories form chiral ribbons. Sci Rep 2013; 3: 1664.

31 Crha I, Zakova J, Huser M, Ventruba P, Lousova E et al. Digital holographic microscopy in human sperm imaging. J Assist Reprod Gen 2011; 28: 725-729.

32 Memmolo P, Di Caprio G, Distante C, Paturzo M, Puglisi R et al. Identification of bovine sperm head for morphometry analysis in quantitative phase-contrast holographic microscopy. Opt Exp 2011; 19: 23215-23226.

33 Katz DF, Dott HM. Methods of measuring swimming speed of spermatozoa. J Reprod Fertil 1975; 45: 263-272.

34 Edwards C, Arbabi A, Popescu G, Goddard LL. Optically monitoring and controlling nanoscale topography during semiconductor etching. Light Sci App/ 2012; 1: e30; doi:10.1038/lsa.2012.30.

(C) This work is licensed under a Creative Commons Attributioncc) this license, visit http://creativecommons.org/licenses/by-nc-nd/3.0 